\title{
Research on the Application of Swarm Intelligence Technology in the Field of Satellite Clusters
}

\author{
Niu Jianjun ${ }^{+}$, Shi Shengwei, Jin Xue, Liu Ye and Zhao Zeya \\ Beijing Institute of Tracking and Telecommunication Technology, China
}

\begin{abstract}
The satellite cluster technology and developments are presented in this paper. The main swarm intelligence algorithms and latest progress in this field are discussed. The Future applications and research issues on swarm intelligence algorithms in satellite clusters are proposed and discussed further in detail in this paper. The proposals in this paper would promote the development of satellite cluster and multi-spacecraft technology.
\end{abstract}

Keywords: satellite cluster, swarm intelligence, space application

\section{Introduction}

With continuous development of the space technology, the application of large-scale microsatellite clusters would become one of the main directions of space science research in the future for performing complex space tasks [1] [2] [3]. The urgent needs of the autonomous control of satellite and swarm intelligence technology application emerged due to the large number of micro-satellites, fast-changing dynamic environment and cluster formation. The technology would overcome disadvantages of traditional ground-based centralized control algorithms, time control inefficiency, management space and time management constrains. The technology enhances the overall intelligence and performance capacity of satellites clusters. Subsequently it will upgrade the space technology to provide technological reserve and support.

\section{Satellite Cluster Technology}

Artificial satellites have been widely used in scientific exploration and research, meteorology, communications, navigation, resource surveying, and military affairs. To improve the service capability of satellite systems, the traditional approach of increasing the payload to achieve a functional expansion has resulted in increasing sizes of satellites and increasing higher costs for developing and launching satellites. Further, it leads to significantly higher task risk. As satellite design advances towards miniaturization and microminiaturization, satellite formations and constellations are undergoing rapid development [4] [5]. Microsatellites are small in size and have simple functionality. A formation of multiple microsatellites working collaboratively can accomplish the same tasks as a large individual satellite and even some functions that would be difficult for the large satellite. By changing the structure of the formation, microsatellite clusters can also execute diverse tasks.

Satellite formations can reduce the cost of satellite development and maintenance, while improving the overall reliability of a satellite system. The European Space Agency's (ESA's) CLUSTER II project in 2000 used four identical satellites to observe the structure and dynamics of the plasma boundary layer between the Earth and the Sun [6]. In 2015, the National Aeronautics and Space Administration (NASA) launched the

Corresponding author. Tel.: + 8613717717108 .

E-mail address: niu_jj@sina.com. 
Magnetospheric Multiscale Mission, which contained four satellites, to achieve high-precision measurement of the Earth's electromagnetic field via ion spectrometry [7]. In 2015, the Laser Interferometer Space Antenna(LISA) project, comprising three satellites, was jointly initiated by NASA and ESA and realized the observation of time fluctuations in general relativity [8]. Sun Yat-sen University in China undertook the Tianqin Project, which is estimated to take 15 to 20 years to detect a gravitational-wave signal using six low-earth orbit (LEO) satellites [9]. The Taiji Project initiated by the Chinese Academy of Sciences plans to launch three satellites into solar orbit by 2033 to detect and investigate the space gravitational waves [10].

The use of microsatellites to build satellite constellations, creating satellite networks and providing services, has recently become a research hotspot. OneWeb, a US-based company, will launch a total of 720 LEO satellites before 2027, adopting the satellite cluster approach to build a global high-speed broadband network [11]. The StarLink Project of the American company SpaceX plans to launch around 12,000 satellites by 2025 to provide high-speed internet services to ground terminals by direct connection to the satellites [12]. Under this project, the first batch of 60 satellites was launched in May 2019. China has announced the Hongyun Project with the aim of building a space-based broadband Internet comprising 156 satellites by 2022 for providing high-definition voice services and low-latency data services [13]. The Galaxy Project, initiated by Galaxy Space, plans to launch thousands of 5G satellites to form a network constellation, seamlessly expand the ground-communication network, and realize full coverage of land, sea, and sky, thus enabling users to access high-speed and flexible 5G networks [14]. The Hongyan (swan goose) Global Satellite Constellation Communication System operated by the China Aerospace Science and Technology Corporation intends to launch around 300 small satellites by 2020 to provide global real-time data communication and integrated information services [15].

Satellite formations and constellations essentially form clusters, which comprise several satellites that accomplish tasks by mutual collaboration. Because of the relatively long distance between satellites themselves and between satellites and the Earth, as well as the rapid changes in their positions, it is difficult to maintain communication links. The traditional centralized control method will create heavy communication burden and may even lead to communication paralysis, resulting in the failure of the overall task. To ensure the successful implementation of a task, the satellites must have the capabilities of autonomous operations and control, adopting distributed collaborative control and operation.

\section{Swarm Intelligence Technology}

Considering birds, ants, fish, bees, wolves, or many other animals in nature, an individual of the species will only have limited independent cognition; however, by adjusting their behavior through simple information exchange, a swarm can quickly search for food and avoid natural enemies, thus reflecting a strong vitality and swarm intelligence. A swarm intelligence optimization algorithm is a type of stochastic optimization algorithm derived by simulating the behavior of natural swarms in the process of long-term evolution, such as predation, escape from enemies, and reproduction. Major examples of such algorithms include Ant Colony Optimization (ACO), which simulates the behavior of ants searching for food; Particle Swarm Optimization (PSO), which simulates the foraging behavior of birds; Cultural Algorithm (CA), which simulates the evolution of human society; Artificial Fish-Swarm Algorithm (AFSA), which simulates the living habits of fish; and Artificial Bee Colony algorithm (ABC), which simulates the behavior of bees when searching for food.

Marco Dorigo proposed the ACO algorithm in 1991 [16]. In the process of searching, ants in a swarm can release and perceive "pheromones," and consequently form a positive feedback mechanism, which is the basis for them to choose a route. Ultimately, this positive feedback process enables almost all ants to find the shortest path. ACO can resolve the traveling salesman problem, combinatorial optimization problem, and many engineering problems.

In 1994, Robert G. Reynolds developed CA [17], which simulates the evolution of human society using a double-evolutionary mechanism comprising a swarm space and a belief space, which are two relatively independent evolutionary processes; the swarm space transfers the individual experience formed during the process of evolution to the belief space, whereas the belief space acquires, stores, and optimizes the swarm experience, such that the swarm has better functionality and a higher speed of evolution. 
In 1995, J. Kennedy and R. C. Eberhart proposed the PSO algorithm [18], which simulates the foraging behavior of birds. In PSO, based on the observation of the foraging activities of birds, a simplified model is established by considering the speed and position of birds when they fly. Leveraging the sharing of individual information in the swarm, the movement of the entire swarm evolves in the solution space; thus, an optimal solution can be achieved in this manner. The PSO algorithm is widely used in various optimization problems owing to its simplicity and fast convergence speed.

In 2002, Li Xiaolei proposed AFSA [19], which is derived from the adaptive behavior of individual fish, since they tend to find a location rich in nutrients in a water body and then gather in swarms. By constructing a simple model of the primary behavior of individuals and achieving local optimization, the optimal solution is ultimately obtained for the swarm. The entire algorithm does not require either centralized control or prior knowledge about the problem; it is a swarm intelligence algorithm with a strong adaptive capacity.

In 2002, Xie Xiaofeng presented the Social Cognitive Optimization (SCO) algorithm [20], which was derived from the fact that human society develops by theoretical learning. As human intelligence accumulates through the learning process, human beings eventually develop stronger knowledge-learning processes by observing each other's behavior and the consequences thereof, especially the behavior of the objects that stimulate them, than those of other general biological swarms. Because an individual's learning iterates through observation and imitation, the SCO algorithm has a fast convergence speed. It has resolved some problems in the fields of nonlinear and multi-objective optimization, as well as some engineering problems.

In 2005, Karaboga et al. proposed the ABC algorithm [21], derived from the honey-gathering activities of bees. In the ABC algorithm, the behavior of individual bees is divided into recruitment and abandonment. The simple behavior of a single bee drives the swarm to demonstrate complex behavior and ultimately obtains the optimal solution. The ABC algorithm has a fast convergence speed and is widely used in optimization problems.

Other swarm intelligence optimization methods include Cuckoo Search (CS), Bacterial Foraging Optimization (BFO), and Glowworm Swarm Optimization (GSO) [22]. In general, CS algorithm has virtues of simpler model and wider universality, but it is slower in convergence speed and is easy to fall into local optimization; BFO algorithm has good parallelism and is difficult to fall into the local optimum, however, it has slower convergence speed and less accuracy; while GSO algorithm has stronger global search ability and simpler process, but its convergence is unstable[23].

In addition, F. Van Den Bergh et al. proposed a co-evolutionary PSO algorithm in 2001. In this algorithm, different particle swarms evolve independently and achieve co-evolution through information sharing. Wang Xuefei et al., inspired by the small-world-network model, proposed a novel PSO algorithm with dynamic topology in 2007. In 2015, Jin Yaochu proposed a social-learning PSO algorithm, which introduced a social learning mechanism into PSO, whereby each particle can learn from any other particle in the current swarm with a greater fitness value [24].

\section{Application Analysis}

Swarm intelligence is characterized by strong adaptability, distribution, self-organization, decentralization, and consistent coordination. Swarm intelligence algorithms are widely used for collaborative control of unmanned aerial vehicle (UAV) formation. The successful adoption of swarm intelligence technology in projects such as the autonomous fleet of Roland University, Hungary and the Collaborative Operations in Denied Environment program and low-cost UAV swarming technology of the US Army[25]. The UAV formations demonstrated better collaboration and intelligence advantages than artificial systems. Motorola laboratory and European Communications Organization adopted swarm intelligence technology to propose a distributed spectrum assignment algorithm, which showed good extendibility and environmental adaptability [26].

The model of satellite cluster system adopting swarm intelligence technology is illustrated in Figure 1. The swarm intelligence technology can be applied to small satellite groups composed of several satellites; It 
could also optimization the behavior of the whole satellite cluster through information exchange between small satellite groups.

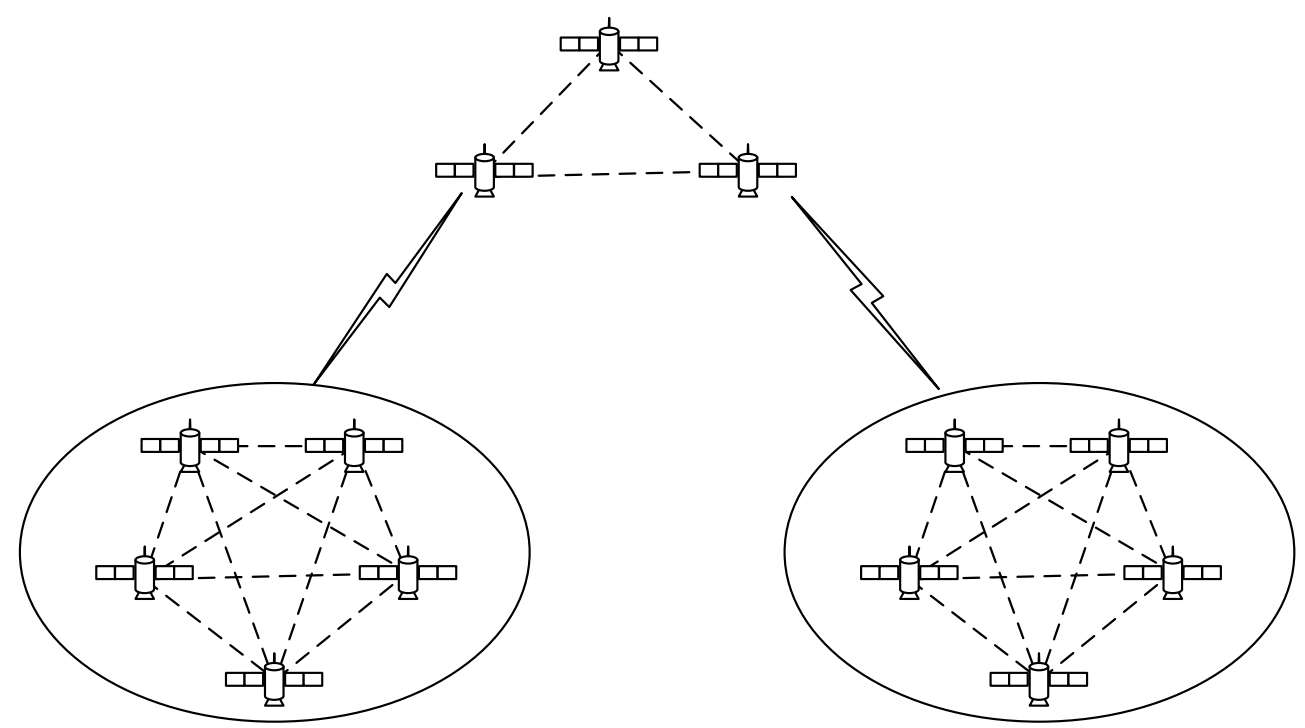

Fig. 1: Model of Satellite Cluster System Adopting Swarm Intelligence Technology

Moreover, the use of swarm intelligence technology for distributed collaborative control for a large number of dynamic satellite clusters has broad application prospects.

\subsection{Satellite Communication}

As satellites and other spacecraft acquiring more and more information, the amount of information that needs to be transmitted is increasing rapidly. Satellite communication is characterized by wide coverage, large communication capacity, fast topological change, and a high bit error rate [27] [28]. Dong Yi et al. proposed a wavelength assignment technology based on ACO for a distributed satellite optical network [29] and investigated large-scale distributed satellite network communication. In terms of dynamic changes in the data transmission routing caused by the continuous movement of satellites and changes in the space environment, other types of swarm intelligence can also be used to solve the complex routing issues to achieve better network performance and improve the convergence speed of the routing algorithm afterwards. In addition, approaches such as the optimization of communication frequency and communication time can also be used to increase the information capacity and transmission rate of satellite communication.

\subsection{Earth Observation}

Earth observation satellites usually include remote-sensing, electronic reconnaissance, ocean surveillance, missile early-warning, and other satellites, which can execute all-round observations on land, ocean, and in the atmosphere from space [30]. Among these, remote-sensing satellites can photograph ground observations, whereas electronic reconnaissance satellites can receive signals from radiation sources in space, thus determining the parameters and geographical location of a target [31]. Moreover, earth observation from space has advantages in terms of a wide reconnaissance range, strong mobility, and fast travel speed; however, it also has some limitations, including a long repeated reconnaissance cycle and short effective reconnaissance time spent on the same target or area [32]. To overcome such limitations during earth observation, swarm intelligence technology can be applied to facilitate a satellite cluster's general performance through intelligent collaborative observation. For point or area targets, different spacecraft can be judiciously arranged to conduct observations over different periods. Collaborative observation can also be made according to the satellites' side-swaying ability, amplitude, and orbit. By optimizing the scheduling of satellite resources, the collaborative-intelligence reconnaissance capability of multiple satellites, efficiently combining general and detailed observations and the target positioning accuracy will be impoved. In addition, the observation and reconnaissance coverage can also be expanded and the reconnaissance time can be extended. 


\subsection{Task Planning}

Satellite task planning is the process of scheduling resources and operations related to satellite tasks. In general, it is necessary under certain constraints to assign tasks to spaceborne resources and determine the start-stop time of various operations and activities [33]. Sensor resources on satellites are often limited; consequently, when it is necessary to track or observe multiple targets, sensor scheduling becomes an NP-hard problem [34]. Existing satellite resources will also reveal weaknesses, such as poor real-time information acquisition ability and insufficiently sustained support during emergency tasks [35]. Spacecraft task planning is a process of flight control under resource-constrained conditions, and flight control operation is a typical knowledge-processing procedure. Considering the above problems, swarm intelligence technology can also be adopted to integrate satellite resources under constrained conditions. Sensors could be reasonably allocated to various targets to reduce resource conflicts between different tasks, maximize users’ requirements, and thereby improve the overall reconnaissance level of the system.

\subsection{Formation Networking}

When performing space tasks, satellite formations and constellations usually exhibit special geometric structures; moreover, there are certain accuracy requirements in terms of their relative positions in space. In addition, it is necessary to prevent collisions between satellites and other flying objects [4]. Traditional navigation-based orbit determination and the combined-orbit determination by a space-ground-based network involve significant participation by ground stations. In the future, once a large number of satellites form a cluster, controlling them from earth-based stations will be difficult due to the huge number of control objects and the complex space environment. When large-scale satellites or other spacecraft perform Moon, Mars, or outer-space explorations, they will be far away from the ground station, so that space perturbations, asteroids, and other factors will have a greater impact on their operation; thus, the formation structure and networking of satellite swarms will be difficult to manage through ground-based control. Because formation network maintenance represents a nonlinear dynamic model, achieving autonomous orbit determination between satellites is a difficult and important topic. Swarm intelligence optimization algorithms can be used in the fields of formation networking and maintenance to improve the adaptive autonomous-control ability of satellites and multiple spacecraft. In addition, this could allow management and control of large-scale microsatellite clusters, such as independent operation control management, collision avoidance, and rapid networking.

\section{Conclusion}

With the miniaturization of satellites and the continuous development of their perception and computing capabilities, there has been a gradual trend of satellite cluster or multiple spacecraft working collaboratively to complete complex tasks. Multi-satellite collaboration is a type of optimization problem in essence. NASA has applied advanced swarm intelligence technology to the task-planning system of the autonomous nanotechnology swarm (ANTS). It plans to launch 1000 ANTS pico-satellites to explore asteroids in the asteroid belt by 2030 and solve the problems associated with the unpredictable space environment [36]. Research on the application of swarm intelligence technology to satellite cluster differs from usual research in this field. Because satellites and other spacecraft are constrained by size, energy consumption, and space radiation [37], the algorithm application would inevitably need to be considered under various restrictive conditions. In addition, the algorithm requires multiple verifications, development, and optimization depending on the use of the satellites.

Space serves as a comprehensive stage for the application of new technologies, and the application of swarm intelligence to the field of space will definitely push forward space exploration in an unmanned and intelligent direction.

\section{References}

[1] British news: The United States Launched a Satellite Cluster Foe Earth Observation. Xinhua News Agency. Jan. 30, 2019.

[2] Russia expands satellite cluster by increasing 156 satellites to enhance space force. Cankaoxiaoxi.com, Aug. 8, 
2019.

[3] Z. Yue. The French Pléiades neo satellite group will reshape the global earth observation market, Infrared. 2017,38 (10): 36 .

[4] S. Hu, X. Wang, and L. Liu L. Overview on flight issues of satellite constellation and formation. Progress in Astronomy. 2003, 21 (3): 231-240.

[5] F. Du, G. Li, H. Zhu, and F. Yun. Overview of the development of the next generation small satellite constellation communication system. Satellite Applications. 2015, (5): 14-19.

[6] B. Gao, and W. Jiao. Master of space weather observation-Cluster-2 satellite. Space International. 2000, (12): $17-22$.

[7] R. Garner. http://www.nasa.gov/mission_pages/mms/overview/i ndex.html. National Aeronautics and Space Administration. 2017.12.6.

[8] Z. Guo, R. Cai, and Y. Zhang. Gravitational wave detection: A new era of gravitational wave astronomy. Science \& Technology Review. 2016, 34(3): 30-33.

[9] Z. Liu. 'Tianqin Project' captures cosmic ripple in space. Science and Technology Review. 2016, 34 (3): 53-54.

[10] Z. Liu, Y. Piao, and C. Qiao. Multi-band gravitational wave cosmic research and space Taiji project. Modern Physics. 2016, (5): 28-33.

[11] M. Mark, Holmes, and T. Lan. OneWeb will ultimately conquer the global connectivity problem. Satellite and Network. 2017, 30-35.

[12] S. Zhang. Essence, technology and competition: Discussion about starlink constellation. Satellite and Network. 2018, 18-20.

[13] China aerospace science and technology corporation plans to launch 156 small satellites to create a space-based internet. China Youth Daily. Aug. 30, 2017.

[14] Homepage of galaxy space, http://www.yinhe.ht, Mar. 2020.

[15] The launch of "'Hongyan” ' makes mobile phone signal available everywhere. Invention and Innovation. 2018, (4): 22.

[16] A. Colorni, M. Dorigo, and V. Maniezzo. Distributed optimization by ant colonies. Proc. of the 1st European Conference on Artificial Life, 1991, pp. 134-142.

[17] R. G. Robert. An introduction to cultural algorithms. In: A. V. Sebalk et al. Proc. Of the 3th annual Conf. on Evolution Programming. World Scientific Publishing, 1994. pp. 131-136.

[18] J. Kennedy, and R. Eberhart. Particle swarm optimization. Proc. of IEEE International Conference of Neural Networks. 1995, 1942-1948.

[19] X. Li, Z. Shao, and J. Qian. An optimization model based on animal autonomy: Artificial fish-swarm algorithm. System Engineering Theory and Practice. 2002, (11): 32-38.

[20] X. Xie, W. Zhang, and Z. Yang. Social cognitive optimization for nonlinear programming problems. Proc. of the 1st International Conference on Machine Learning and Cybemetics. Beijing, 2002.

[21] D. Karaboga, and B. Basturk. A powerful and efficient algorithm for numerical function optimization: Artificial bee colony algorithm. Journal of Global Optimization. 2007, 39 (3): 459-471.

[22] S. Lin, C. Dong, M. Chen, F. Zhang, and J. Chen. Overview of new swarm intelligence optimization algorithms. Computer Engineering and Applications. 2018, 54 (12): 1-9.

[23] Lin shijie, Dong Chen, Chen Mingzhi, ZHANG Fan, CHEN Jinghui. Computer Engineering and Applications. 54(12),:1-9, 2018.

[24] R. Cheng, and Y. Jin. A social learning particle swarm optimization algorithm for scalable optimization. Information Sciences. 2015, 291: 43-60.

[25] B. Hai, and P. Li. UAV swarm control based on biological swarm behavior. Science and Technology Herald. 2017 , 35(7): 17-25.

[26] J.C. Dunat, D. Grandblaise, and C. Bonnet, Collaborative allocation of orthogonal frequency division multiplex 
sub-carriers using the swarm intelligence, Journal of Communications, 2006, 1(1): 68-76.

[27] J. Feng, Y. Zhang, and C. Pan. Research on strategies for optimizing channel transmission characteristics of satellite communication networks. Computer Simulation. 2017, 34(2): 230-233.

[28] Y. Wang, J. Fu, J. Zheng, and F. Zou. Comprehensive application mode of satellite communication anti-jamming technology. National Defense Science and Technology. 2018, 39(4): 63-68.

[29] Y. Dong, S. Zhao, Y. Li, J. Zhao, and B. Deng. Research on wavelength assignment technology based on ant colony optimization for distributed satellite optical network. Journal of Electronics and Information Technology. 2015, 37(11): 2650-2656.

[30] X. Geng, Z. Zhang, and J. Zhu. Overview of the status of global earth observation satellites and related industrial development. Digital Communication World. 2009, 72-75.

[31] G. Yang, X. Liu, and J. Guo. Analysis on application and advantages of multi-satellite collaborative reconnaissance of electronic reconnaissance satellites. Aerodynamic Missile Journal. 2018, (1): 58-61.

[32] G. Wang, and X. Li. Analysis on intelligence reconnaissance capabilities of electronic reconnaissance satellites. Aerospace Electronic Warfare. 2016, 32(6): 44-48.

[33] P. Wang, and Y. Tan. A brief overview of satellite task planning for earth observation. Application Research of Computers. 2008, 25(10): 2893-2897.

[34] X. Huang, Y. Deng, X. Hu, and Y. Yuan. Satellite scheduling method based on chessboard encoding particle swarm optimization. Computer Engineering and Design. 2013, 34(1): 307-313.

[35] C. He, X. Zhu, and D. Qiu. Multi-satellite collaborative scheduling method for emergency imaging observation task. System engineering and electronics. 2012, 34(4): 726-731.

[36] X. Wen, S. Zhu, and F. Zhang. Analysis and prospect concerning future NASA group satellite system. Aerospace China. 2012, (3): 34-36.

[37] Y. Hou, and M. Ni. Research and implementation of satellite cluster distributed technology. Computer Systems and Applications. 2017, 26(12): 233-239. 